\title{
Kernel Principal Component Regression in Reproducing Kernel Hilbert Space
}

\author{
Chooleewan DACHAPAK*, Shunshoku KANAE*, Zi-Jiang YANG*, Kiyoshi WADA* \\ *Department of Electrical and Electronic System Engineering, Kyushu University \\ Fukuoka, 812-8581 JAPAN \\ Tel: +81-92-642-3958; Fax:+81-92-642-3939; \\ E-mail:\{joe, czjin, yoh, wada\}@dickie.ees.kyushu-u.ac.jp
}

\begin{abstract}
In this study, we proposed Kernel Principal Component Analysis (KPCA) which is applied for feature selection in a high-dimensional feature space which is nonlinearly mapped from an input space by a Gaussian kernel function. By using Mercer Kernels, we can compute principal components in a high dimensional feature space. Then, the extracted features are employed as preprocessing step for an ordinary least squares regression in the feature space which is Reproducing Kernel Hilbert Space (RKHS).
\end{abstract}

\section{Introduction}

In the present study, we are interested in the feature selection method as the appropriate preprocessing step for an ordinary least squares regression, since the suitable feature selection can increase the overall performance of algorithms [1]. Kernel Principal Component Analysis is based on the computation of the standard linear PCA in the feature space mapped from input space by some nonlinear function [2]. We have applied KPCA method for feature selection in a high dimensional or possibly infinite feature space $F$ (with dimension $M \leq \infty)$. This allows us to extract the nonlinear principal components up to the number of data points $n(n \leq M)$. We compute a dot product in the feature space by means of kernel functions in input space, i.e. $k(\boldsymbol{x}, \boldsymbol{y})=(\Phi(\boldsymbol{x}) . \Phi(\boldsymbol{y}))$, and we never need the mapped patterns $\Phi(x)$ explicitly. It is often the case that a small number of Kernel Principal Component is sufficient to account for most of the structure in the data. Also, the Kernel PCs are uncorrelated each other, and it is possible that KPCA can decrease the effect of noise [2]. Then, the features extracted by KPCA method are employed as a regressors of the ordinary least squares regression. This means it performs a linear regression in the feature space $F$.

\section{Kernel Principal Compo- nent Analysis}

First, mapping the data nonlinearly into the feature space $F$ by the nonlinear function as following,

$$
\Phi: \boldsymbol{R}^{N} \rightarrow F, \quad \boldsymbol{x} \mapsto \boldsymbol{X} .
$$

Note that the feature space $F$ could have an arbitrarily large, possibly infinite, dimensionality [3]. Assume that our data mapped into feature space is centered, $\sum_{k=1}^{n} \Phi\left(x_{k}\right)=0$. Then, in $F$ space, a covariance matrix can be expressed as

$$
\bar{C}=\frac{1}{n} \sum_{j=1}^{n} \Phi\left(x_{j}\right) \Phi\left(x_{j}\right)^{T} .
$$

Define $(n \times M)$ matrix $\Phi$,

$$
\Phi^{T} \triangleq\left[\begin{array}{llll}
\Phi\left(x_{1}\right) & \Phi\left(x_{2}\right) & \ldots & \Phi\left(x_{n}\right)
\end{array}\right] .
$$

So $(M \times M)$ matrix $\bar{C}$ can be rewritten as

$$
\bar{C}=\frac{1}{n} \Phi^{T} \Phi .
$$

Let $(n \times n)$ matrix $K$ denote

$$
K=\boldsymbol{\Phi} \Phi^{T} .
$$

Noting $n \bar{C}$ and $K$ 's nonzero eigenvalues are the same. Let $\lambda_{k}, V^{k}$ be $\bar{C}$ 's $k^{t h}$ eigenvalues and eigenvectors respectively, where $k=1,2, \ldots, P_{F} . P_{F}$ is the number of nonzero eigenvalues. $P_{F} \leq n$.

$$
n \lambda_{k} \boldsymbol{V}^{k}=n \bar{C} \boldsymbol{V}^{k}=\boldsymbol{\Phi}^{T} \boldsymbol{\Phi} \boldsymbol{V}^{k} .
$$

Pre-multiply $\Phi$,

$$
n \lambda_{k} \boldsymbol{\Phi} \boldsymbol{V}^{k}=\boldsymbol{\Phi} \boldsymbol{\Phi}^{T} \boldsymbol{\Phi} \boldsymbol{V}^{k}=K \boldsymbol{\Phi} \boldsymbol{V}^{k} .
$$


Let $\gamma^{k}$ be

$$
\gamma^{k}=\Phi V^{k}
$$

then,

$$
K \gamma^{k}=\tilde{\lambda} \gamma^{k}
$$

Note that $\tilde{\lambda}_{k}=n \lambda_{k}$. Moreover from,

$$
\Phi^{T} \gamma^{k}=\Phi^{T} \Phi V^{k}=\tilde{\lambda}_{k} \boldsymbol{V}^{k}
$$

so we can obtain

$$
\boldsymbol{V}^{k}=\frac{1}{\tilde{\lambda}_{k}} \Phi^{T} \boldsymbol{\gamma}^{k}
$$

Since it is required that eigenvector $V$ in $F$ space must be normalized as the following condition,

$$
\boldsymbol{V}^{k^{T}} \boldsymbol{V}^{k}=1
$$

This translates into a normalization condition,

$$
\begin{aligned}
1 & =\frac{1}{\tilde{\lambda}_{k}^{2}} \gamma^{k^{T}} \Phi \Phi^{T} \gamma^{k}=\frac{1}{\tilde{\lambda}_{k}^{2}} \gamma^{k^{T}} K \gamma^{k} \\
& =\frac{1}{\tilde{\lambda}_{k}^{2}} \gamma^{k^{T}} \tilde{\lambda}_{k} \gamma^{k}=\frac{1}{\tilde{\lambda}_{k}} \gamma^{k^{T}} \gamma^{k} .
\end{aligned}
$$

From the above condition, we obtain

$$
\boldsymbol{V}^{k}=\frac{1}{\sqrt{\tilde{\lambda}_{k}}} \Phi^{T} \gamma^{k}
$$

Let $\alpha^{k}$ be

$$
\alpha^{k}=\frac{1}{\sqrt{\hat{\lambda}_{k}}} \gamma^{k}
$$

Then eigenvector $\boldsymbol{V}^{k}$,

$$
\boldsymbol{V}^{k}=\Phi^{T} \alpha^{k} .
$$

Finally, project $\Phi(x)$ onto the $k^{\text {th }}$ nonlinear principal component by the dot product between $\boldsymbol{V}^{k}$ and $\Phi(x)$ as below,

$$
\boldsymbol{V}^{k} . \Phi(x)=\alpha^{k^{T}} \Phi \Phi(x) .
$$

The above $1^{s t}, \ldots, P_{F}^{t h}$ nonlinear principal components, $\boldsymbol{\Phi} V$, are employed as a new uncorrelated regressors for the regression model in the feature space. We used only first $p$ components in order to reduce the noise effect since the components whose variances are very small can be assumed as the variance from noise.

\section{Kernel Principal Compo- nent Regression}

Consider the standard regression model in the feature space

$$
\boldsymbol{y}=\Phi \xi+\epsilon,
$$

where $y$ is a vector of $n$ observations, $\Phi$ is an $(n \times M)$ matrix of regressors whose $i^{\text {th }}$ row is the vector $\Phi\left(x_{i}\right)$ of the mapped $x_{i}$ observation into $M \leq \infty$ dimensional feature space $F, \xi$ is a vector of regression coefficients and $\epsilon$ is a vector of error terms. We assume that regressors are centered. Now, let $\left(M \times P_{F}\right)$ matrix $V$ denote

$$
V=\left[\begin{array}{llll}
V^{1} & V^{2} & \ldots & V^{P_{F}}
\end{array}\right]
$$

then,

$$
\Phi^{T} \Phi V=V \Lambda
$$

Note that $\Lambda$ is an diagonal matrix of $\left\{\tilde{\lambda}_{1}, \tilde{\lambda}_{2}, \ldots, \tilde{\lambda}_{P_{F}}\right\} . \quad V$ is an orthogonal matrix so the linear regression model can be expressed in the term of new regressors as

$$
\begin{gathered}
y=\Phi V V^{T} \xi+\epsilon=B w+\epsilon . \\
B=\Phi V, \quad w=V^{T} \xi .
\end{gathered}
$$

Here we can see that the new regressors $B$ are in the term of Kernel Principal Component which are the extracted feature we are interested in. And

$$
\boldsymbol{B}^{T} \boldsymbol{B}=V^{T} \Phi^{T} \Phi V=V^{T} V \Lambda=\Lambda
$$

So the least square estimator of coefficient $w$ can be expressed as

$$
\hat{\boldsymbol{w}}=\left(\boldsymbol{B}^{T} \boldsymbol{B}\right)^{-1} \boldsymbol{B}^{T} \boldsymbol{y}=\Lambda^{-1} \boldsymbol{B}^{T} \boldsymbol{y} .
$$

From the above estimator expression, we can see that it is difficult to find $\boldsymbol{B}$ directly. The easier way to find $B$ by using kernel function will be shown. Let $y_{k}$ be the $k^{t h}$ component of $y$ and $\epsilon_{k}$ be the $k^{t h}$ component of $\epsilon$, from the linear regression model, we obtain

$$
y_{k}=\Phi\left(x_{k}\right)^{T} \boldsymbol{\xi}+\epsilon_{k}=\Phi\left(x_{k}\right)^{T} V \boldsymbol{w}+\epsilon_{k}
$$

Here, consider $V^{T} \Phi\left(x_{k}\right)$, from the definition of $V$, we can see that

$$
V^{T} \Phi\left(x_{k}\right)=\left[\begin{array}{c}
V^{1^{T}} \Phi\left(x_{k}\right) \\
V^{2^{T}} \Phi\left(x_{k}\right) \\
\vdots \\
V^{M^{T}} \Phi\left(x_{k}\right)
\end{array}\right]=\left[\begin{array}{c}
\beta_{1}\left(x_{k}\right) \\
\beta_{2}\left(x_{k}\right) \\
\vdots \\
\beta_{M}\left(x_{k}\right)
\end{array}\right] .
$$

Note that $\beta_{i}\left(x_{k}\right)=\boldsymbol{V}^{i^{T}} \Phi\left(x_{k}\right)$, because $\boldsymbol{V}^{i}$ and $\boldsymbol{\alpha}^{i}$ have the relationship as

$$
V^{i}=\Phi^{T} \alpha^{i}
$$

so $\beta_{i}\left(\boldsymbol{x}_{k}\right)$ can be represented by

$$
\beta_{i}\left(x_{k}\right)=\alpha^{i^{T}} \Phi \Phi\left(x_{k}\right)
$$




$$
=\alpha^{i^{T}}\left[\begin{array}{c}
\Phi\left(x_{1}\right)^{T} \Phi\left(x_{k}\right) \\
\Phi\left(x_{2}\right)^{T} \Phi\left(x_{k}\right) \\
\vdots \\
\Phi\left(x_{n}\right)^{T} \Phi\left(x_{k}\right)
\end{array}\right]=\alpha^{i^{T}}\left[\begin{array}{c}
k\left(x_{1}, x_{k}\right) \\
k\left(x_{2}, x_{k}\right) \\
\vdots \\
k\left(x_{n}, x_{k}\right)
\end{array}\right]
$$

We compute the dot products $\Phi\left(x_{i}\right)^{T} \Phi\left(x_{k}\right)$ in the feature space by means of kernel functions in input space $k\left(\boldsymbol{x}_{i}, \boldsymbol{x}_{k}\right)=\Phi\left(\boldsymbol{x}_{i}\right)^{T} \Phi\left(\boldsymbol{x}_{k}\right)$. Moreover, there is advantage that we do not need to map $\Phi(\boldsymbol{x})$ explicitly through out the study. Then, define $k\left(x_{k}\right)$ as following,

$$
\boldsymbol{k}\left(\boldsymbol{x}_{k}\right)=\left[\begin{array}{c}
k\left(\boldsymbol{x}_{1}, \boldsymbol{x}_{k}\right) \\
k\left(\boldsymbol{x}_{2}, \boldsymbol{x}_{k}\right) \\
\vdots \\
k\left(\boldsymbol{x}_{n}, \boldsymbol{x}_{k}\right)
\end{array}\right]
$$

then $\beta_{i}\left(\boldsymbol{x}_{k}\right)$ becomes

$$
\beta_{i}\left(x_{k}\right)=\alpha^{i^{T}} k\left(x_{k}\right) .
$$

So

$$
\begin{aligned}
V^{T} \Phi\left(x_{k}\right)=\left[\begin{array}{c}
\beta_{1}\left(x_{k}\right) \\
\beta_{2}\left(x_{k}\right) \\
\vdots \\
\beta_{P_{F}}\left(x_{k}\right)
\end{array}\right] & =\left[\begin{array}{c}
\alpha^{1^{T}} \\
\alpha^{2^{T}} \\
\vdots \\
\alpha^{P_{F} T}
\end{array}\right] k\left(x_{k}\right) \\
& =A^{T} k\left(x_{k}\right) .
\end{aligned}
$$

Note that

$$
A=\left[\begin{array}{llll}
\alpha^{1} & \alpha^{2} & \ldots & \alpha^{P_{F}}
\end{array}\right] .
$$

We can rewrite (25) as

$$
y_{k}=k\left(x_{k}\right)^{T} A w+\epsilon_{k}=k\left(x_{k}\right)^{T} \theta+\epsilon_{k}
$$

where

$$
\theta=A w=A V^{T} \xi .
$$

From the definition of $B$,

$$
\begin{aligned}
B & =\Phi V \\
B & =\left[\begin{array}{c}
\Phi\left(x_{1}\right)^{T} \\
\Phi\left(x_{2}\right)^{T} \\
\vdots \\
\Phi\left(x_{n}\right)^{T}
\end{array}\right] V=\left[\begin{array}{c}
\Phi\left(x_{1}\right)^{T} V \\
\Phi\left(x_{2}\right)^{T} V \\
\vdots \\
\Phi\left(x_{n}\right)^{T} V
\end{array}\right] \\
B & =\left[\begin{array}{c}
k\left(x_{1}\right)^{T} \\
k\left(x_{2}\right)^{T} \\
\vdots \\
k\left(x_{n}\right)^{T}
\end{array}\right] A,
\end{aligned}
$$

where,

$$
\begin{aligned}
& {\left[\begin{array}{c}
k\left(x_{1}\right)^{T} \\
k\left(x_{2}\right)^{T} \\
\vdots \\
k\left(x_{n}\right)^{T}
\end{array}\right]} \\
& =\left[\begin{array}{cccc}
k\left(x_{1}, x_{1}\right) & k\left(x_{2}, x_{1}\right) & \cdots & k\left(x_{n}, x_{1}\right) \\
k\left(x_{1}, x_{2}\right) & k\left(x_{2}, x_{2}\right) & \cdots & k\left(x_{n}, x_{2}\right) \\
\vdots & \vdots & \ddots & \vdots \\
k\left(x_{1}, x_{n}\right) & k\left(x_{2}, x_{n}\right) & \cdots & k\left(x_{n}, x_{n}\right)
\end{array}\right]=K .
\end{aligned}
$$

We can rewrite $B$ as

$$
B=K A \text {. }
$$

So we can formulate the KPCR model as following expression,

$$
y=B w+\epsilon=K A w+\epsilon=K \theta+\epsilon .
$$

We prefer (37) to (35) since we can compute $B$ by means of kernel functions in input space without mapping $\Phi(x)$ explicitly. As we mentioned above, we employed the first $p$ nonlinear principal components to create a KPCR model in order to decrease the effect of noise and eliminate large variances of the estimate due to multicollinearities. In this case the first $p$ nonlinear principal components mean the first $p$ column vectors of $A$.

\section{Mercer Kernels}

Kernel functions allow to map the data into the high dimensional feature space. In practice the kernel $k$ is usually defined directly, thus implicitly defining the map $\Phi$ and the feature space $F$ [4]. Clearly, from the symmetry of the inner product, a kernel must be symmetric:

$$
k(\boldsymbol{x}, \boldsymbol{y})=k(\boldsymbol{y}, \boldsymbol{x})
$$

and also satisfy the Cauchy Schawartz inequality:

$$
k^{2}(\boldsymbol{x}, \boldsymbol{y}) \leq k(\boldsymbol{x}, \boldsymbol{x}) k(\boldsymbol{y}, \boldsymbol{y}) .
$$

However, this is not sufficient to guarantee the existence of a feature space. It needs to satisfy Mercer theorem conditions as following.

Proposition 1 If $k$ is a continuous symmetric kernel of a positive integral operator $K$, i.e.

$$
(K f)(\boldsymbol{y})=\int_{C} k(\boldsymbol{x}, \boldsymbol{y}) f(\boldsymbol{x}) d \boldsymbol{x}
$$

with

$$
\int_{c \times c} k(x, y) f(x) f(y) d x d y \leq 0
$$


for all $f \in L^{2}(C)$ ( $C$ being a compact subset of $\boldsymbol{R}^{N}$ ), it can be expanded in a uniformly convergent series (on $C \times C$ ) in terms of Eigenfunctions $\psi_{j}$ and positive Eigenvalues $\lambda_{j}$

$$
k(\boldsymbol{x}, \boldsymbol{y})=\sum_{j=1}^{M} \lambda_{j} \psi_{j}(\boldsymbol{x}) \psi_{j}(\boldsymbol{y})
$$

where $M \leq \infty$.

Note that originally proven for the case where $C=[a, b]$, this proposition also holds true for general compact spaces.

From above, it is straightforward to construct a map $\Phi$, mapping into a potentially infinite dimensional $l^{2}$ space, which does the job. For instance, we may use

$$
\Phi: x \mapsto\left(\sqrt{\lambda_{1}} \psi_{1}(x), \sqrt{\lambda_{2}} \psi_{2}(x), \ldots\right) .
$$

We thus have following result:

Proposition 2 If $k$ is a continuous kernel of a positive integral operator(conditions as in Proposition 1), one can construct a mapping $\Phi$ into a space where $k$ acts as a dot product,

$$
(\Phi(\boldsymbol{x}) \cdot \Phi(\boldsymbol{y}))=k(\boldsymbol{x}, \boldsymbol{y}) .
$$

Gaussain radial basis function kernels is also suggested,

$$
k(\boldsymbol{x}, \boldsymbol{y})=\exp \left(-\frac{\|\boldsymbol{x}-\boldsymbol{y}\|^{2}}{2 \sigma^{2}}\right)
$$

\section{RKHS}

The feature space that $\Phi$ maps into is a Reproducing Kernel Hilbert Space (RKHS). RKHS is a Hilbert space of function $f$ on some set $C$ such that all evaluation functionals $f \mapsto f(y)(y \in C)$ are continuous. In that case, by the Riesze representation theorem, for each $y \in C$ there exists a unique function of $\boldsymbol{x}$, call it $k(\boldsymbol{x}, \boldsymbol{y})$, such that

$$
f(\boldsymbol{y})=\langle f, k(., \boldsymbol{y})\rangle
$$

(here, $k(.,(\boldsymbol{y}))$ is the function on $C$ obtained by fixing the second argument of $k$ to $y$ and $\langle.,$.$\rangle is the$ dot product of the RKHS). In view of this property, $k$ is called a reproducing kernel.

Note that by (45), $\langle f, k(., y)\rangle=0$ for all $\boldsymbol{y}$ implies that $f$ is identically zero. Hence the set of functions $\{k(., y): y \in C\}$ spans the whole RKHS. The dot product on the RKHS thus only needs to be defined on $\{k(., y): y \in C\}$ and can then be extended to the whole RKHS by linearity and continuity. From (45), it follows that in particular

$$
\langle k(., \boldsymbol{x}), k(., \boldsymbol{y})\rangle=k(\boldsymbol{y}, \boldsymbol{x})
$$

for all $x, y \in C$ (this implies that $k$ is symmetric). Note that this means that any reproducing kernel $k$ corresponds to a dot product in another space.

To establish a connection to the dot product in a feature space $F$, we next assume that $k$ is a Mercer kernel . First note that it is possible to construct a dot product such that $k$ becomes a reproducing kernel for a Hilbert space of functions

$$
\begin{aligned}
f(x) & =\sum_{i=1}^{\infty} a_{i} k\left(x, x_{i}\right) \\
& =\sum_{i=1}^{\infty} a_{i} \sum_{j=1}^{M} \lambda_{j} \psi_{j}(x) \psi_{j}\left(x_{i}\right) .
\end{aligned}
$$

Using only linearity, which holds for any dot product $\langle.,$.$\rangle , we have$

$$
\begin{aligned}
& \langle f, k(., y)\rangle \\
& =\sum_{i=1}^{\infty} a_{i} \sum_{j=1}^{M} \lambda_{j} \psi_{j}\left(\boldsymbol{x}_{i}\right)\left\langle\psi_{j}, \psi_{n}\right\rangle \lambda_{n} \psi_{n}(\boldsymbol{y}) .
\end{aligned}
$$

Since $k$ is a symmetric kernel, the $\psi_{i}(i=1, \ldots, M)$ can be chosen to be orthogonal with respect to the dot product in $L^{2}(C)$. Hence it is straightforward to construct a dot product $\langle.,$.$\rangle such that$

$$
\left\langle\psi_{j}, \psi_{n}\right\rangle=\delta_{j n} / \lambda_{j}
$$

(using the Kronecker symbol $\delta_{j n}$ ), in which case (48) reduces to the reproducing kernel property (45).

To write $\langle.,$.$\rangle as a dot product of coordinate vec-$ tors, we thus only need to express the functions of the RKHS in the basis $\left(\sqrt{\lambda_{n}} \psi_{n}\right), n=1, \ldots, M$, which is orthonormal with respect to $\langle.,$.$\rangle , i.e.$

$$
f(x)=\sum_{n=1}^{M} \alpha_{n} \sqrt{\lambda_{n}} \psi_{n}(x) .
$$

To obtain the coordinates $\alpha_{n}$, we compute, using (49),

$$
\begin{aligned}
\alpha_{n} & =\left\langle f, \sqrt{\lambda_{n}} \psi_{n}\right\rangle \\
& =\left\langle\sum_{i=1}^{\infty} a_{i} \sum_{j=1}^{M} \lambda_{j} \psi_{j}\left(x_{i}\right) \psi_{j}, \sqrt{\lambda_{n}} \psi_{n}\right\rangle \\
& =\sqrt{\lambda_{n}} \sum_{i=1}^{\infty} a_{i} \psi_{n}\left(x_{i}\right) .
\end{aligned}
$$

Comparing (50) and (42), we see that $F$ has the structure of a RKHS in the sense that for $f$ and $g$ given by (50) and

$$
g(x)=\sum_{j=1}^{M} \beta_{j} \sqrt{\lambda_{j}} \psi_{j}(x),
$$


we have

$$
(\alpha \cdot \beta)=\langle f, g\rangle \text {. }
$$

Note, moreover, that due to (50), we have $f(x)=$ $(\alpha \cdot \Phi(x))$ in $F$. Comparing to (45), this shows that $\Phi(\boldsymbol{x})$ is nothing but coordinate representation of the kernel as a function of one argument.

\section{Experiment}

In this present study, we employed Gaussian kernel function, $k(\boldsymbol{x}, \boldsymbol{y})=e^{-\left(\frac{\| \boldsymbol{x}-\boldsymbol{y}_{\|^{2}}}{L}\right)}$, where L determines the width of the Gaussian function. We utilized the noisy Chaotic Mackey-Glass Time-Series applying to KPCR method which the regressors are extracted by Kernel PCA. This KPCR method was trained to predict the value at time $t+85$ from inputs at time $t, t-6, t-12, t-18$. The training data partitions were constructed over 3000 training samples in steps of 500 samples. So we got six partitions. The simulations were repeated for the width $L$ from range $(0.2 \hat{\sigma}, 20 \hat{\sigma})$, where $\hat{\sigma}$ is the variance of the overall clean training set, using the step size 0.01. A fixed test set of size 500 data points was employed through out the experiments. The performance of the regression models to predict a clean Mackey-Glass time series was evaluated in terms of the normalized root mean squared error (NRMSE). The best result on the test set averaged over all individual runs are summarized in Table 1.

Table 1 The comparison of the approximation errors (NRMSE) for prediction of Mackey-Glass training set. N/S represents the ratio between the standard deviation of the added Gaussian noise and the underlying time-series.

\begin{tabular}{|c|c|c|c|}
\hline Method & $N / S=0.0$ & $N / S=0.11$ & $N / S=0.22$ \\
\hline$K P C R$ & 0.038 & 0.307 & 0.443 \\
\hline
\end{tabular}

\section{Conclusions}

The Kernel PCA method has been employed for the feature extraction and noise reduction in the preprocessing step for regression problem.

With KPCR method, there are some problems occurred when we remove some Kernel principal components with small eigenvalues in order to reduce the noise present because those Kernel principal components may have a significant contribution for the prediction.

\section{References}

[1] R. Rosipal, M. Girolami, L.J. Trejo, and A. Cichocki. Kernel PCA for Feature Extraction and
De-noising in Non-linear Regression. Neural Computing \& Applications, 10(3), 2001.

[2] B. Schölkopf, C.J.C. Burges, and A.J. Smola. Advances in Kernel Methods Support Vector Learning. The MIT Press, Cambridge, Massachusetts, London England, 1998.

[3] B. Schölkopf, A.J. Smola, and K.R. Muller. Nonlinear Component Analysis as a Kernel Eigenvalue Problem. Neural Computation, 10:1299-1319, 1998.

[4] M.G. Genton. Classes of Kernels for Machine Learning : A Statistics Perspective. Journal of Machine Learning Research, pages 299-312, $2(2001)$.

[5] R. Rosipal and L.J. Trejo. Kernel Partial Least Squares Regression in Reproducing Kernel Hilbert Space. Journal of Machine Learning Research, pages 97-123, December 2(2001).

[6] B. Schölkopf. Support Vector Learning. $P h D$ thesis, Universitat Berlin, Berlin, Germany, 1997.

[7] I.T. Jolliffe. Principal Component Analysis. Springer-Verlag, New York, 1986.

[8] H.D. Vinod and A. Ullah. Recent Advances in Regression Methods. Marcel Dekker,INC., 1981. 

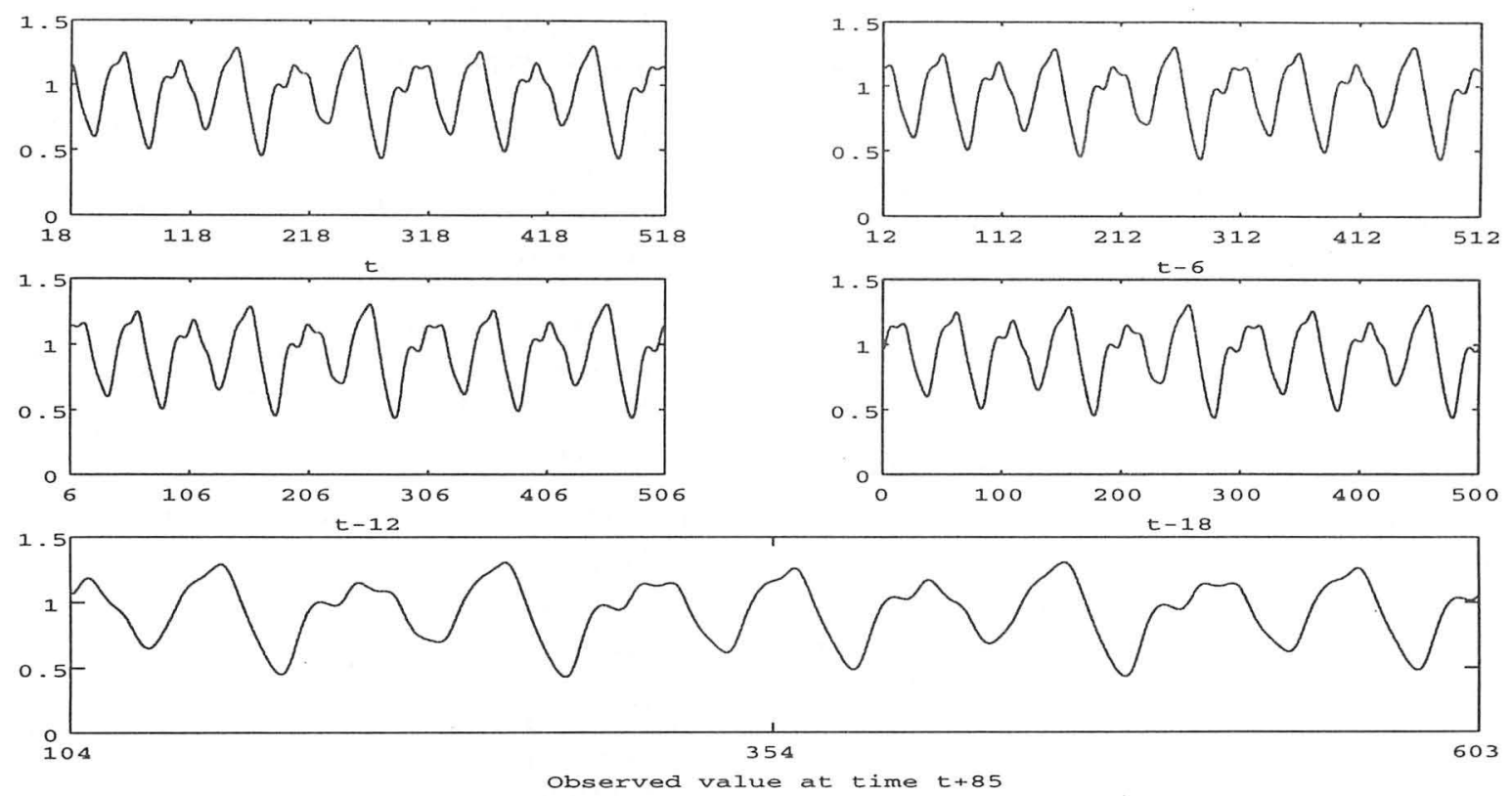

Figure 1: Training data set: 500 samples from 3000 samples of Mackey-Glass time series. First 4 figures show the inputs at time $t, t-6, t-12, t-18$ from $t=19$ to 518 . They are employed as the data set $x$ which are trained by KPCR method to predict the value at time $t+85$ (The bottom shows the observed value at time $\mathbf{t}+85)$.
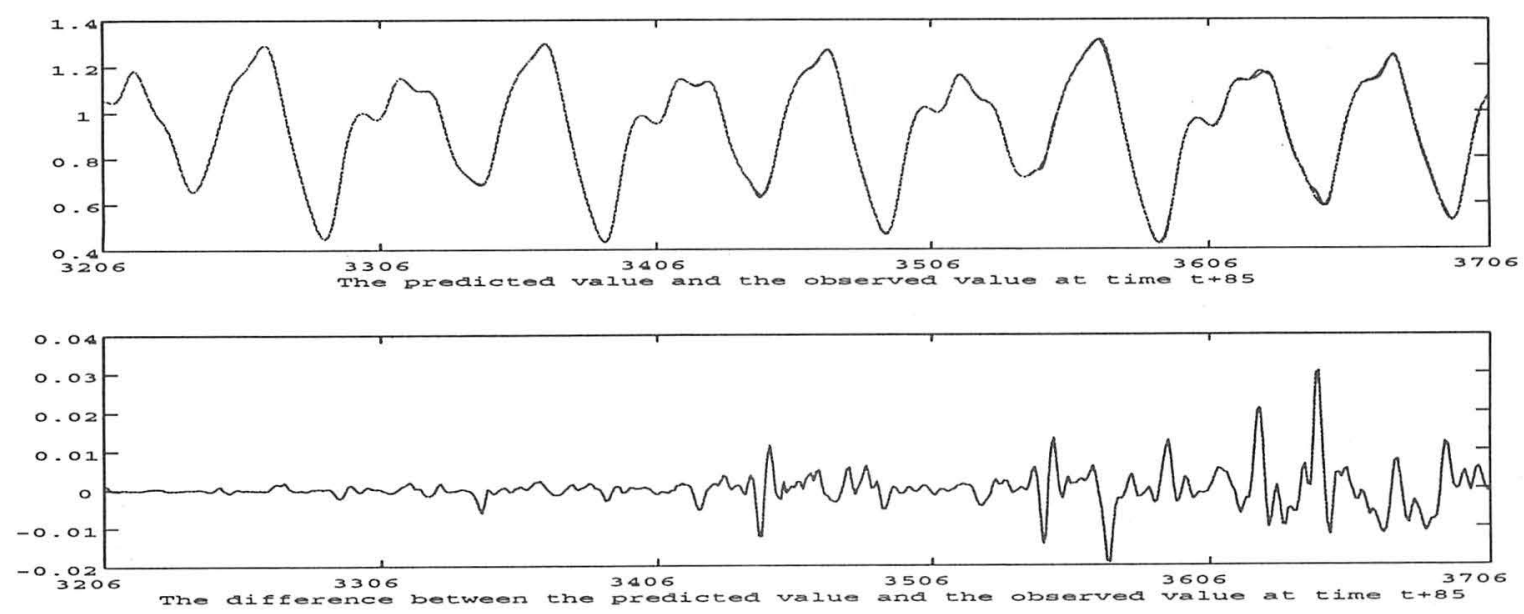

Figure 2: Testing data set: the above shows the predicted value and the observed value at time $t+85$ ( $t=3122$ to 3621 ). And the bottom shows the different value between the predicted value and the observed value. 